\title{
An Optimization Problem Algorithm for Kinematic Design of Mechanisms for Two-Finger Grippers
}

\author{
Chiara Lanni ${ }^{*}$ and Marco Ceccarelli*
}

LARM: Laboratory of Robotics and Mechatronics, DiMSAT- University of Cassino, Italy

\begin{abstract}
In this paper, an analysis of mechanisms in two-finger grippers has been discussed to formulate an optimum design procedure. The design problem has been approached and formulated as a new optimization problem by using fundamental characteristics of grasping mechanisms. In particular, in order to optimize a mechanism for two-finger gripper, an original multi-objective optimum algorithm has been used by considering four different objective functions, such as grasping index, encumbrance of grasping mechanism, acceleration and velocity for finger gripper with respect to the imposed working area. A case study has been reported by using an 8R2P linkage for a proposed two-finger gripper mechanism. Numerical example has been computed to show the soundness of the proposed new optimum design procedure by referring to computational and practical results.
\end{abstract}

Keywords: Grippers, grasp mechanics, mechanisms, analysis, design, experimental validation.

\section{INTRODUCTION}

A gripper is an important component of industrial robots because it interacts with the environment and objects, which are grasped for manipulative tasks. Usually, a gripper of industrial robots is a specialized devise, which is used to grasp one or few objects of similar shape, size, and weight in repetitive operations [1-3].

The manipulative operations are usually performed by using two-finger grippers, which are powered and controlled for the grasping action by one actuator only, $[4,5]$. In addition, two-finger grippers are used both for manipulation and assembling purposes since most of these tasks can be performed with a two-finger grasp configuration $[6,7]$.

Since a gripper gives a great contribution to practical success of using an automated and/or robotized solution, a proper design may be of fundamental importance. The design of a gripper must take into account several aspects of the system design together with the peculiarities of a given application or a multi-task purpose. Strong constraints for the gripping system can be considered for lightness, small dimensions, rigidity, multi-task capability, simplicity and lack of maintenance. These design characteristics can be achieved by considering specific end-effectors or grippers. In the last case a two-finger gripper corresponds to the minimum number of fingers and the minimum complexity of a hand.

An early work on gripper designs can be considered the publication by Lundstrom, [1], who described several gripper designs with rigid fingers and flexible fingers, and even vacuum grippers, and magnetic grippers.

*Address correspondence to these authors at the LARM: Laboratory of Robotics and Mechatronics, DiMSAT- University of Cassino, Via Di Biasio 43, 03043 Cassino (Fr), Italy; Tel: +39-0776-2993663;

E-mail: lanni@unicas.it, ceccarelli@unicas.it
Chen, [2], described several mechanisms for different gripper functions. He also classified mechanical grippers according to pair elements used in their construction as linkage, gear and rack, cam, screw, rope and pulley types and miscellaneous. The selection of a particular mechanism is mainly affected by type of actuators to be employed and type of grasping modalities to be used.

Chelpanov and Kolpashnikov, [8], reported a definition and formalization of the basic problems of gripper mechanisms. In particular, a grasping mechanism can be classified into five classes, namely clamping elements; elements for linking the clamping with the executive elements; executive elements; transmission mechanisms between the drive and executive mechanism.

Belfiore and Pennestrì, [9], presented an atlas of 64 linkage-type grippers. For each mechanism one possible functional scheme has been provided for a better understanding of its kinematic properties. The proposed atlas can be useful to designers in the field of robotics.

Several procedures to design grippers have been reported in literature, as for given in refs. [3, 10-23].

In particular, refs. $[3,10]$ a description of several gripper mechanisms has been reported with design conside-rations.

Dwivedi, Sharma and Sharifi, [11], proposed the design of an intelligent gripper. The intelligent gripper has been composed as a combination of the general-purpose gripper mechanism and force/torque sensor mechanism. In particular, a translational-type gripper mechanism has been chosen as the basis of the general purpose-gripper.

Shimoga, [12], reported algorithms to achieve dexterity primarily to solving an unconstrained linear programming problem where an objective function can be chosen to represent one or more of the currently known dexterity measures for grippers. 
Salunkhe, Mao, and Tasch, [13], developed a mathematical formulation for robust and high quality grasp. In particular, the grasping quality has been obtained by minimizing the entropy of the finger normal force whereas the robustness is ensured by minimizing the perturbations in finger contact locations.

Dubey, Crowder and Chappell, [14], formulated an optimal control of fingertip force during grasping operation. In particular, a controller that is based on fuzzy logic has been considered in order of performing optimal stable grasp of objects without knowing their mass and frictional properties and with a minimum applied force.

Hester, Cetin, Kapoor and Tesar, [15], used multiple performance criteria both at the finger and hand levels to generate a preliminary grasp and then an optimum grasp.

Osyczka and Krenich, [16], described a new genetic algorithm for solving nonlinear multi-criterion optimization problem. In the proposed method the tournament selection has been considered as the core of the procedure.

Penisi, Carbone and Ceccarelli, [17], developed an optimum design procedure and validation testing for mechanisms of two-finger grippers. In particular, an optimization problem has been formulated by taking into account both the kinematics and statics of the gripper action.

Ceccarelli, Cuadrado and Dopico, [18], presented a simple and efficient procedure for optimum dimensional synthesis of grasping mechanisms. The proposed design has been based on a suitable formulation of grasping performance of grasping mechanisms by using natural coordinates.

Krenich, [19], formulated a design optimization problem of robot grippers by taking into account six objective functions and several constraints.

Ceccarelli, [20, 21], proposed an optimum design for grasping mechanism of two-finger gripper in the form of a suitable optimization problem by defining a grasping index, which takes into account some fundamental characteristics of the grasp action.

Zheng and Qian, [22], formulated an optimal grasp planning and dynamic force distribution in multi-fingered grasping.

Lanni, [23], studied the design problem for two-finger gripper by considering the numerical and experimental characterization as regarding with the impact during a grasp. In particular, in order to optimize a mechanism for twofinger gripper, a multi-objective optimum algorithm has been used by considering four different objective functions, such as grasping index, encumbrance of gripper mechanism, acceleration and velocity for finger gripper with respect to the imposed working area.

In this paper, performance criteria are investigated and a design problem has been approached and formulated as an optimization problem by using the basic characteristics of grasping mechanisms. A suitable algorithm has been developed for a general optimum design of grasping mechanisms.

A study has been reported as a numerical example in the paper to show the soundness of the proposed optimum design procedure by referring to computational and practical results.

\section{MECHANISMS FOR GRIPPERS}

Among all the problems encountered in designing robots, the most crucial one concerns with the end-effector. Basic features for a gripper depend strongly of the grasping mechanism. Thus, factors can be considered before choosing a grasping mechanism as following:

- Characteristics of the gripper, which include maximum payload, dimensions, orientations, number of the composed links;

- Characteristics of the objects, which include weight, body rigidity, nature of material, geometry, dimensions, condition, position and orientation, contact surfaces, forces acting on the object and environmental conditions;

- Gripper technology, for the construction of components (mechanism links and finger parts) with proper manufacturing and materials;

- Flexibility of the gripper, whether it allows rapid replacement, or easy adjust and external modification, or adaptation to a family of objects that are contained within a range of specifications;

- Cost for design, production and application to robot operation and maintenance.

In fact, those characteristics are fundamental from a practical viewpoint for the grasping purpose, since they may describe the range of exerting force on the object by the fingers, the size range of the objects which may be grasped and a particular manipulation type. Thus, a dimensional design of gripper mechanisms may have great influence on the maximum dimensions of the grasped object by a gripper, and on the grasping force, since the mechanism size may affect the grasp configuration and transmission characteristics. These peculiarities can be considered well known when it is taken into account the great variety of mechanisms which have been used.

The basic components of a two-finger gripper are given in ref. [21], (Fig. 1): fingers are the elements that execute the grasp on objects; finger tips are directly in contact with a grasped object; grasping mechanism is the transmission component between the actuator and the fingers; actuator is the power source for the grasping action of a gripper.

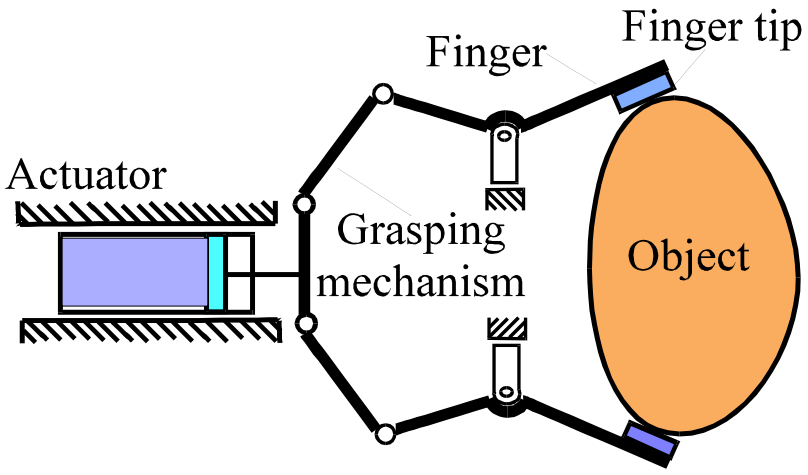

Fig. (1). A scheme for mechanical design for two-finger grippers.

In order to execute a specific grasping task a design problem consists in selecting a proper gripper mechanisms and sizing its kinematic design. 
Many different types of kinematic chains can be used as for example those that are reported and shown in refs. [1-3, $9,11]$. Special attention requires the motion of the fingers. This motion can be linear, rotative or a combination of both. Needs of limited encumbrance, stiff design, light mechanical design and easy operation may limit the number of the mechanism links. In fact, most of industrial gripper mechanisms show kinematic chains that are composed by four-bar, slider-crank linkages as shown in Fig. (2), [2].
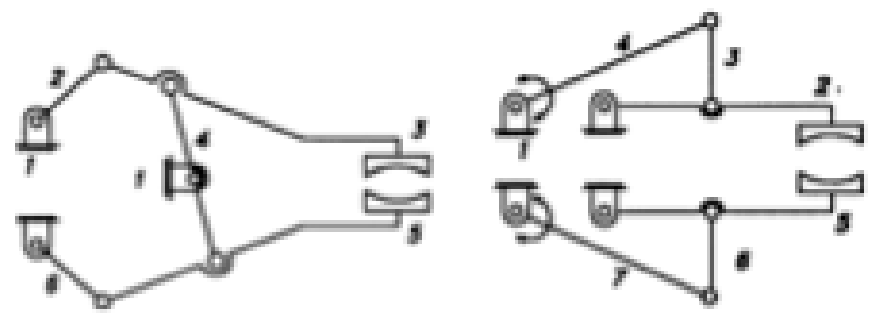

a)
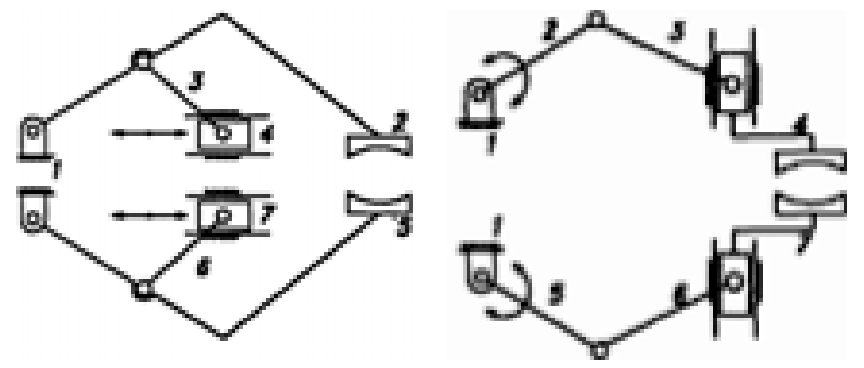

b)

Fig. (2). Examples of grasping mechanism in [2] as based on: a) four-bar linkage; b) slider-crank linkage.

In particular, Fig. (2) shows examples of grasping mechanism in industrial grippers, which are actuated by linear or rotative actuator using revolute and prismatic joints with one or two actuators. The fingers can move with a swinging or a parallel motion. In Fig. (2a) the grasping mechanisms are based on a mechanisms composed by fourbar linkages which are based on revolute joints. The two fingers move with an approximately parallel motion. In Fig. (2b) the grasping mechanisms are based on a mechanisms composed by slider-crank linkages which are based on prismatic and revolute joints. The two fingers move with an approximately linear motion.

Most of industrial grippers are actuated by a linear actuator. However, two actuators can be useful when the fingers can operate independently with a symmetric or unsymmetrical behaviour.

Many others types of gripper mechanisms are used in order to achieve suitable mechanical design with grasping efficiency, small size, robust design, light and low-cost devices.

There are many different types of industrial grippers composed by two-fingers that are implemented in commercial grippers, like those given in refs. [1, 24-27].

Examples of two-finger grippers that are actuated pneumatically are shown from Figs. (3) to (7). In particular, Fig. (3a) shows a view of a commercial industrial 2-jaws parallel-acting self-centering pneumatic gripper that is produced by Gimatic Handling [24]. Fig. (3b) shows the complex mechanical design, corresponding to the gripper in Fig. (3a), in which the identification of the kinematic chain can be very difficult if the gripper is not observed during the operation with and without an object. The mechanical design of the gripper in Fig. (3b) shows how to obtain a parallel motion of the fingers by the chain reported in Fig. (3c).

Fig. (4a) shows a view of commercial industrial 2-jaws self centering radial pneumatic gripper that is produced by Gimatic Handling [24]. Fig. (4b) shows the related mechanical design of a gripper in Fig. (4a). The kinematic scheme of Fig. (4c) emphasize the use of cam system. The cam connection can be very useful to have small-sized designs with an easy understanding of the gripper operation.

Fig. (5a) shows a view of a commercial industrial gripper that is produced by GMG System [25]. Fig. (5b) shows the related mechanical design of a gripper in Fig. (5a). The kinematic scheme of Fig. (5c) shows how is possible to obtain different combination of the motion during opening and closing phases. In fact, depending to the dimensions of the links of the fingers is possible to obtain parallel open / a)

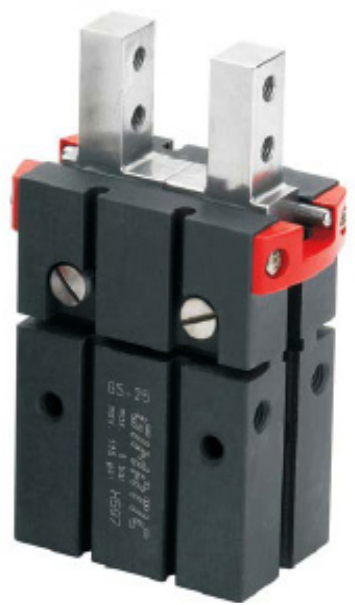

b)

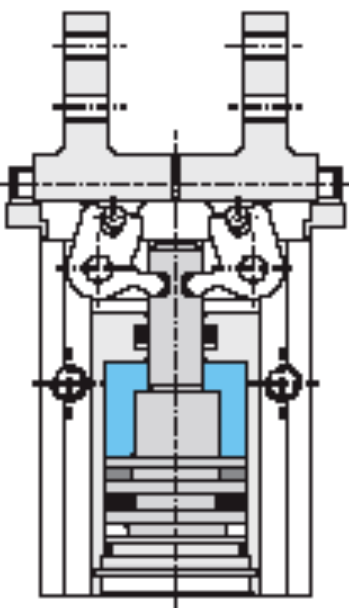

c)

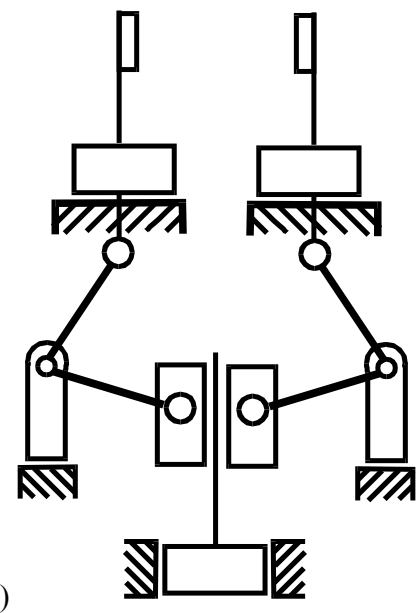

Fig. (3). An industrial 2-jaws parallel-acting self centering pneumatic gripper, [24]: a) a view of GIMATIC GS-25 type; b) a mechanical design; c) a kinematic scheme. 


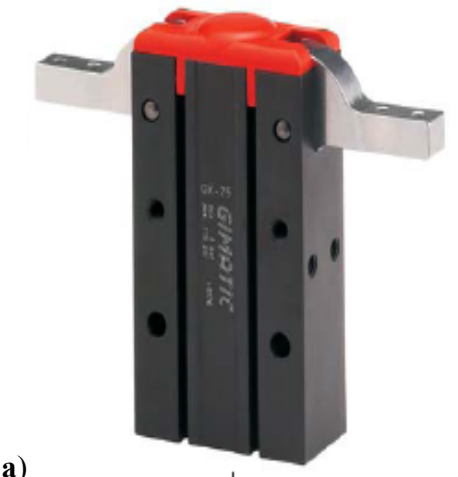

a)

Fig. (4). Industrial 2-jaws self centering radial pneumatic gripper [24]: a) a view of GIMATIC GX-25 type; b) a mechanical design; c) a kinematic scheme.

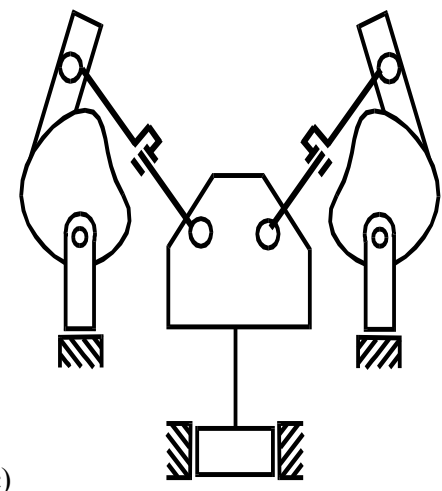

c)

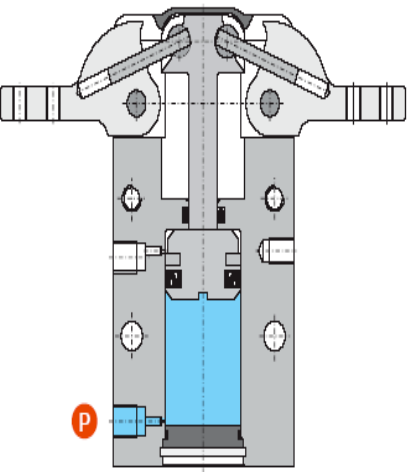

a)

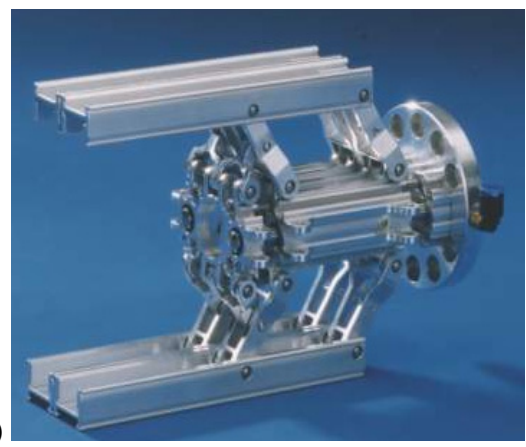

b)

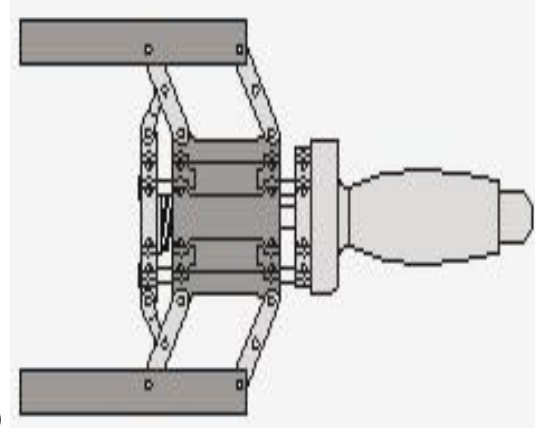

c)

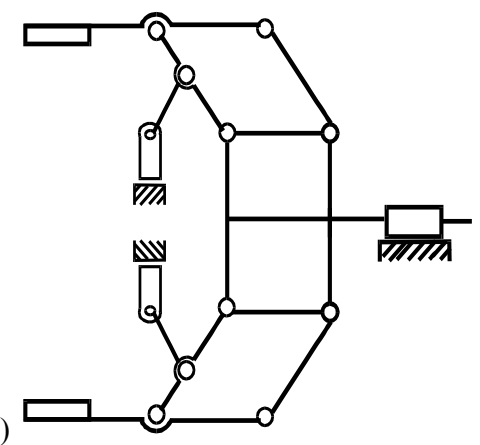

Fig. (5). An industrial gripper, [25]: a) a view of GMG 102 type; b) a mechanical design; c) a kinematic scheme.

parallel close, parallel open/swivelling close, and swivelling open/swivelling close action.

Fig. (6a) shows a view of an industrial toggle gripper that is produced by AGI Components [26]. Fig. (6b) shows the related mechanical design of a gripper in Fig. (6a). The kinematic scheme of Fig. (6c) shows how is possible to obtain the synchronized parallel motion of the fingers as generated by a pinion mechanism that is powered by a double-acting piston. The jaws are supported by a T-SLOT way.
Fig. (7a) shows a view of a commercial industrial toggle gripper that is produced by SMC Components [27]. Fig, (7b) shows the related mechanical design of a gripper in Fig. (7a). The kinematic scheme of Fig. (7c) shows that the moving prismatic joints and rolling contact ensure an approximately circle trajectory for each finger.

\section{DESIGN PROBLEMS}

A fundamental problem both for designing and operating a gripper mechanism can be recognized in the modeling of a)

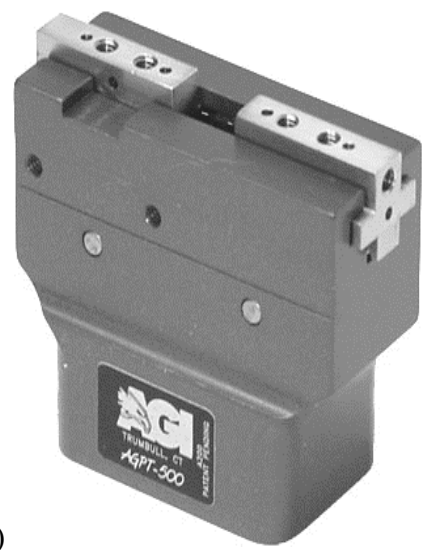

b)

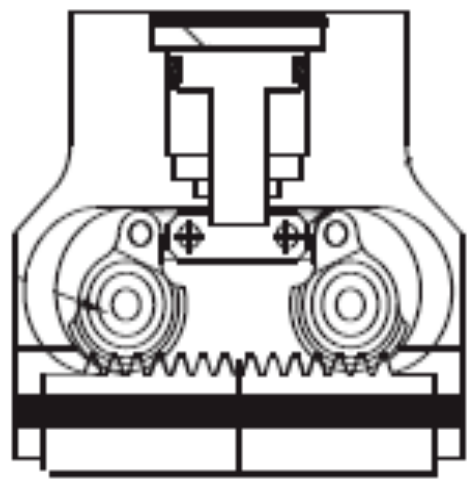

c)

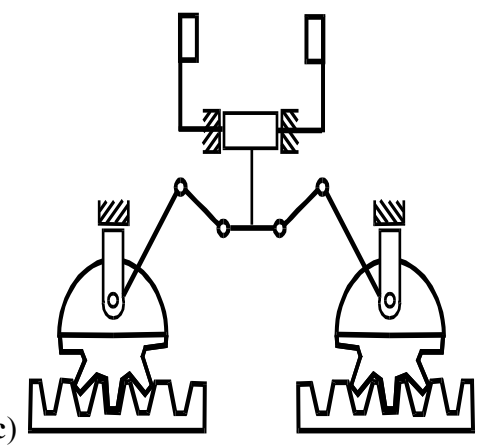

Fig. (6). An industrial toggle gripper, [26]: a) a view of AGI PT500 type; b) a mechanical design; c) a kinematic scheme. 
a)

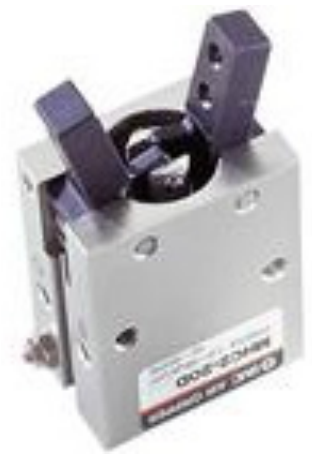

b)

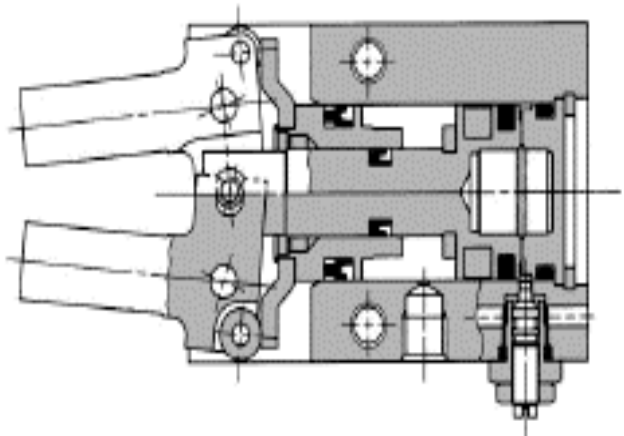

c)

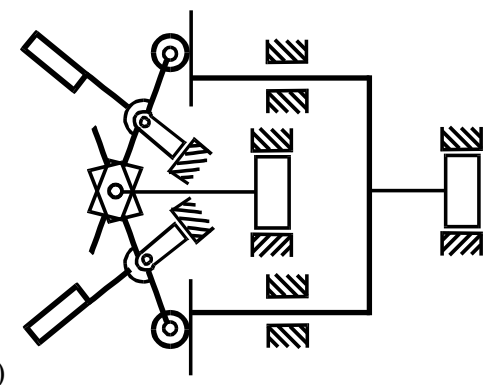

Fig. (7). An industrial toggle gripper, [27]: a) a view of SMC MHC2 type; b) a mechanical design; c) a kinematic scheme.

the basic characteristics of a gripper in order to identify the mechanism chain and its kinematic characteristics but by considering yet the integration with the other components in a Mechatronics design.

The design problem for grippers consists of sizing all the components of a gripper in order to ensure suitable grasping performances for a proper grasping manipulation.

A design procedure can be proposed in the following steps:

1. To determine the basic characteristics of the grasped object, in terms of dimensions, weight, shape, material, density, delicateness;

2. To individuate the environments in which the object should be grasped;

3. To evaluate the required grasping force as a function of maximum dimension of the grasped object;

4. To design finger tips and their sensors;

5. To design a control system;

6. To choose a chain type and to size the gripper mechanism and its operation;

7. To size the actuator by considering the efficiency of the grasping mechanism;

8. To design all the components that are needed to control the gripper operation.

In particular, it is to note that for step 3, the model and formulation for the equilibrium of the grasp can be elaborated in order to evaluate the maximum grasping force. An in-depth analysis can be focused on the interactions among object and fingers. The static equilibrium of a grasped object between the fingers can be expressed by considering all the forces that can be determined along the directions of the contact, squeezing and slipping lines [21].

At step 5, a control system can be designed as depending of the level of grasping force regulation, which is required for the grasping task.

At step 6, a specific kinematic chain can be chosen by a designer by considering atlas, books, catalogs of existing industrial grippers, expert systems for searching, and even designers expertise. The dimensional design of gripper mechanisms can be approached by using traditional techniques for dimensional synthesis of mechanisms. Alternatively, an optimum design of a gripper mechanism can be formulated. In this case, by using commercial computational tools a suitable design procedure can give optimum characteristics for an optimum use of a grasping mechanism as a function of specific grasping purposes.

At step 8, all the components of the control system can be designed for the given gripper tasks. The power circuit and actuator can be pneumatic, hydraulic and electric. Thus, the components of the control system can be sized as function of actuator performances, gripper mechanism and control grasping force.

In general, a design of two-finger grippers can be expressed explicitly through suitable formulations to give an analytical system of design equations which express the relations between all the components.

The design parameters can be summarized as, (Fig. 8):

- $\quad$ Link sizes $1_{i}, i=1,2, \ldots, N$ in which $\mathrm{N}$ indicates the number links;

- Configuration angles $\alpha_{\mathrm{J}}, \mathrm{J}=1,2, \ldots, \mathrm{N}_{\mathrm{g}}$ in which $\mathrm{N}_{\mathrm{g}}$ is the number of joint of gripper mechanism;

- $\quad$ Actuation force Q;

- Characteristics of the control systems in term of $K_{P}$, $\mathrm{K}_{\mathrm{D}}, \mathrm{K}_{\mathrm{I}}$, proportional, derivative and integrative gains, respectively.

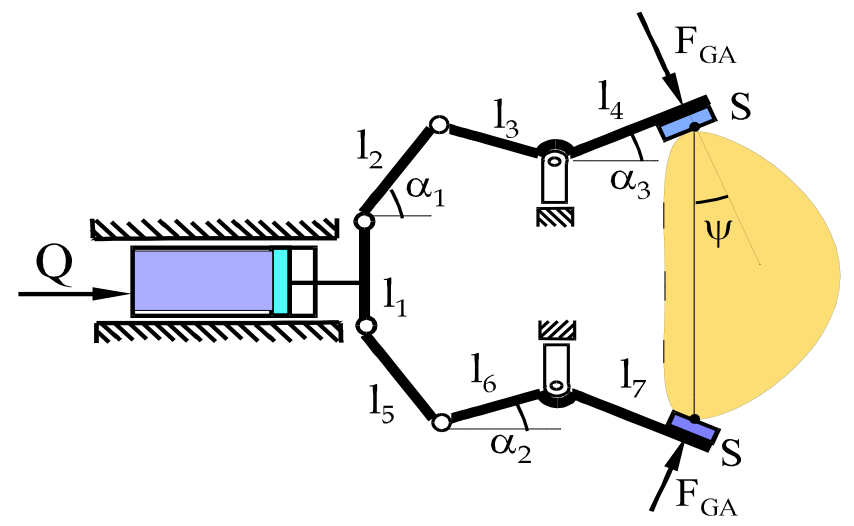

Fig. (8). Design parameters for a two-finger gripper.

\section{A FORMULATION FOR OPTIMUM DESIGN}

The problem of optimum design of mechanisms has quite a long history. In fact, most of these problems have been modelled as non-linear programming problems [28]. 
Similarly, the design problem of grippers can be formulated as a multi-criteria optimization problem in which several criteria can be considered.

An optimum design formulation for gripper mechanisms consists of minimizing an objective function $\mathrm{F}$ that is subject to satisfy constraints, material properties, object characteristics, and peculiarities of the grasping task.

A critical point of such a design formulation is to choose a suitable objective function $\mathrm{F}$, which must include peculiarity aspects of the gripper mechanism and its design parameters in order to obtain solutions with proper optimal performance characteristics.

A design formulation can be expressed in the form of optimization problem in the form of:

$\min \mathbf{F}(\mathbf{x})$

subject to,

$\mathbf{G}_{\mathrm{i}}(\mathbf{x})<\mathbf{0} \quad \mathrm{i}=1, \ldots, \mathrm{k}$

$\mathbf{H}_{\mathrm{i}}(\mathbf{x})=\mathbf{0} \quad \mathrm{i}=1, \ldots, \mathrm{m}$

where $\mathbf{x}=\left[\mathrm{x}_{1}, \mathrm{x}_{2}, \ldots, \mathrm{x}_{\mathrm{n}}\right]$ is the vector of the $\mathrm{n}$ design variables; $\mathbf{F}(\mathbf{x})$ is the vector of objective functions $f_{i}(i=$ $1, \ldots, N)$ that express the optimality criteria, $\mathbf{G}_{\mathbf{i}}(\mathbf{x})$ is the vector of $\mathrm{k}$ inequality constraint functions that describes limiting conditions, and $\mathbf{H}_{\mathbf{i}}(\mathbf{x})$ is the vector of $m$ equality constraint functions that describes the design prescriptions.

The multi-objective function $\mathbf{F}$ can be formulated with computer-oriented algorithms when its components $f_{i}$ are computed numerically through suitable analysis procedures.

Similarly, the constraint functions $\mathbf{G}$ and $\mathbf{H}$ can be formulated by using suitable evaluation of design and operation constraints as well as those additional constraints that are needed for computational issues. Thus, the problem for achieving optimal results from the formulated multiobjective optimization problem consists mainly in two aspects, namely to choose a proper numerical solving technique and formulate the optimality criteria with computational efficiency.

The constraints can be expressed for so many and different characteristics but they should be analytically formulated by considering geometrical characteristics of the grasping mechanisms, forces acting on the joints, maximum and minimum dimensions of the grasped object, minimum and maximum grasping force acting for a specific grasped object, minimum dimensions of the gripper [19].

Indeed, the solving technique can be selected among the many available ones, even in commercial software packages, by looking at a proper fit and/or possible adjustments to the formulated problem in terms of number of unknowns, nonlinearity type, and involved computations for the optimality criteria and constraints. On the other hand, the formulation and computations for the optimality criteria and design constraints can be conceived and performed by looking also at the peculiarity of the numerical solving technique.

Those two aspects can be very helpful in achieving an optimal design procedure that can give solutions with no great computational efforts and with possibility of engineering interpretation and guide. Since the formulated design problem is intrinsically high no-linear, the solution will be obtained when the numerical evolution of the tentative solutions due to the iterative process converges to a solution that can be considered optimal within the explored range. Therefore a solution can be considered an optimal design but as a local optimum in general terms. This last remark makes clear once more the influence of suitable formulation with computational efficiency for the involved criteria and constraints in order to have a design procedure, which is significant from engineering viewpoint and numerically efficient.

\section{OPTIMALITY CRITERIA}

In general, an optimum design procedure can be considered by means the following steps:

1) Identification of design constraints and performance characteristics for a given application;

2) Formulation of basic performances;

3) Analysis of optimality criteria through numerical algorithms;

4) Formulation of a single and/or multi-objective optimization problem for design purposes;

5) Numerical solution of the multi-objective optimization and interpretation of results;

6) Determination of a design solution through a suitable model;

7) Mechanical design of all the components and details.

In this paper, we have addressed attention mainly to the design step 3 that are related with optimality criteria and an optimum design procedure has been proposed using a multiobjective optimization problem in terms of grasping index PI, encumbrance of grasping mechanism, acceleration and velocity of the gripper mechanism.

An optimum synthesis is useful to find the mechanism that has the better efficiency which is variable with the configuration adopted by the mechanism. Thus, the optimum solution should have a small variation of the efficiency in the whole range of objects that can be grasped. In order to satisfy the proposed requirements and to consider an expression for the mechanical efficiency, the grasping index PI can be defined as proposed in [21].

$\mathrm{PI}=\frac{\mathrm{F}_{\mathrm{GA}} \cos \psi}{\mathrm{Q}}$

where $\mathrm{Q}$ indicates the actuating force, $\mathrm{F}_{\mathrm{GA}}$ is the grasping force applied to the contact point $\mathrm{S}$ and $\psi$ is the angle grasp configuration, Fig. (8). The grasping index PI can be evaluated for several gripper mechanisms by using a principle of virtual work. Thus, in order to optimize a gripper mechanism, one can define the following optimality criterion $\mathrm{f}_{1},[23]$.

$\mathrm{f}_{1}=\mathrm{PI}$

The number of the links affects the dimension and weight of the device. Minimizing the dimension of the gripper can be useful to reduce its weight and costs and to provide better 
a)

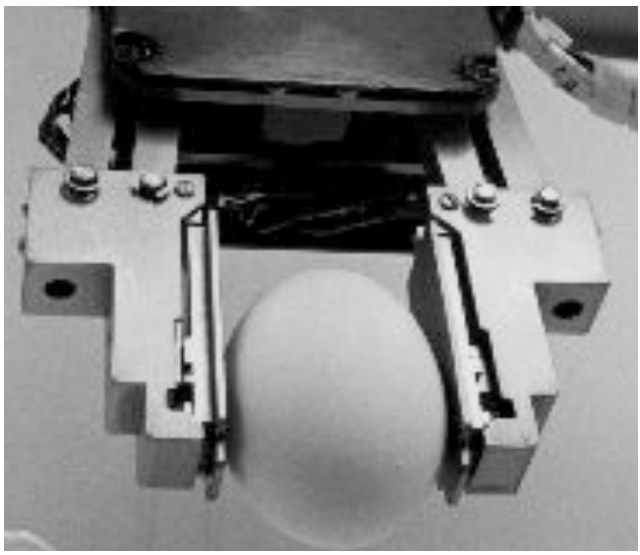

b)

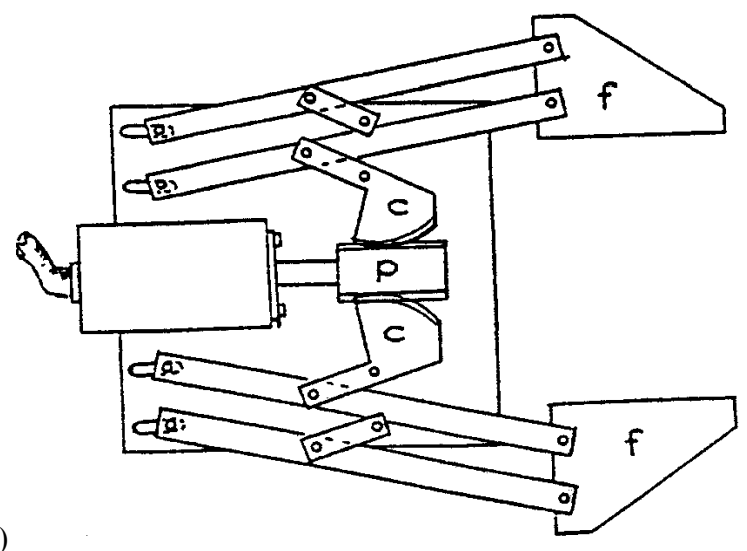

Fig. (9). A commercial industrial gripper, [30]: a) a view of IBM-7565 type; b) a mechanical design [31].

clearances in a specific workstation, [29]. Thus, in order to optimize the gripper mechanism, one can define the following optimality criterion $\mathrm{f}_{2},[23]$

$\mathrm{f}_{2}=\sqrt{\sum_{\mathrm{i}=1}^{\mathrm{N}} \mathrm{l}_{\mathrm{i}}^{2}}$

The acceleration of the gripper should not be too large because it causes inertia phenomenon which may increase the disturbance acting on the grasped object during a grasp. Thus, in order to optimize the gripper mechanism, one can define the following optimality criterion $\mathrm{f}_{3}$, [23]

$\mathrm{f}_{3}=\frac{\mathrm{acc}_{\text {max }}-\mathrm{acc}_{\text {min }}}{\mathrm{acc}_{\text {med }}}$

where $\mathrm{acc}_{\max }, \mathrm{acc}_{\min }$ and $\mathrm{acc}_{\text {med }}$ indicate the maximum, minimum and average acceleration during the grasping action, respectively.

The velocity of the gripper should be constant in order to avoid sudden variation of vibrations and external disturbances during the grasped object. Thus, in order to optimize the gripper mechanism, one can define the following optimality criterion $\mathrm{f}_{4},[23]$.

$$
\mathrm{f}_{4}=\frac{\mathrm{vel}_{\text {max }}-\mathrm{vel}_{\text {min }}}{\mathrm{vel}_{\text {med }}}
$$

where $\mathrm{vel}_{\text {max }}, \mathrm{vel}_{\text {min }} \mathrm{e} \mathrm{vel}_{\text {med }}$ indicate the maximum, minimum and average velocity during the grasping action, respectively.

The purpose of the above proposed formulation is to simplify the required computations and reduce the overall computational cost for gripper optimization by giving also the possibility to a designer to understand and guide the computational evolution in a numerical technique for optimization problem solution.

\section{A NUMERICAL EXAMPLE}

The example refers to an IBM gripper, in Fig. (9), whose gripper mechanism can be modelled as a 8R2P linkage in Fig. (10). A parallel motion of a finger $\mathrm{f}$ is obtained by actuating the 8R2P linkage through a double acting pneumatic piston that is connected to the crank c by a rackpinion gear sector. In particular, in Fig. (10) a link is added to $1_{0}$ to model the rack-pinion gear sector from which the piston actuates the crank $1_{0}$.

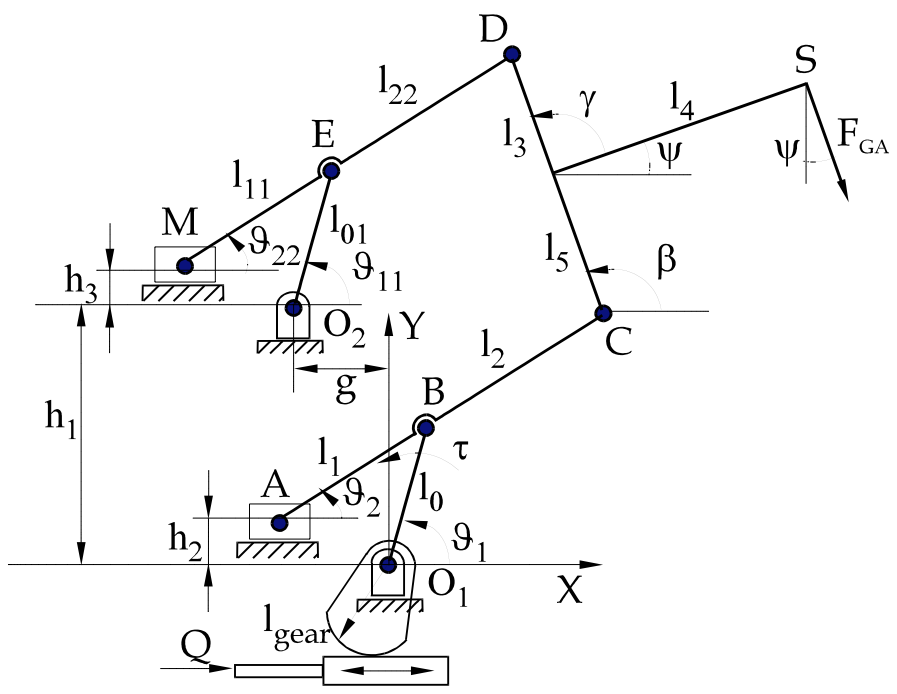

Fig. (10). A kinematic scheme of two-finger gripper mechanism in Fig. (9). 
The analysis of kinematic characteristics of the gripper mechanism can be obtained as referring to a frame $\mathrm{O}_{1} \mathrm{XY}$ in Fig. (10), where $1_{0}, 1_{1}, 1_{01}, 1_{11}, 1_{2}, 1_{22}, 1_{3}$ and $1_{5}$ are the lengths of the links; $1_{4}$ is the finger link length; $\vartheta_{1}$ is the input angle of the crank $1_{0} ; \vartheta_{2}$ is the angle of the crank $1_{1} ; h_{1}$ is the vertical offset between revolute joints; $h_{2}$ and $h_{3}$ are the slider joint offsets; $g$ is the horizontal offset between the frame revolute joints; $\gamma$ is the finger angle between $1_{4}$ and $1_{3}$; $\beta$ is the orientation angle between $1_{2}$ and $1_{5} ; 1_{\text {gear }}$ is the radius of rack-pinion gear sector.

The position, velocity, and acceleration of point $\mathrm{S}$ can be computed by considering the closure equations for $8 \mathrm{R} 2 \mathrm{P}$ linkage as follows. The position of point $\mathrm{S}$ can be evaluated through the expressions

$\mathrm{x}_{\mathrm{S}}=\mathrm{x}_{\mathrm{C}}+\mathrm{l}_{4} \cos (\psi)+1_{5} \cos (\beta)$

$\mathrm{y}_{\mathrm{S}}=\mathrm{y}_{\mathrm{C}}+1_{4} \sin (\psi)+1_{5} \sin (\beta)$

The orientation angles $\beta$ and $\psi$ can be computed by

$\beta=\tan ^{-1}\left(\frac{\mathrm{y}_{\mathrm{D}}-\mathrm{y}_{\mathrm{c}}}{\mathrm{x}_{\mathrm{D}}-\mathrm{x}_{\mathrm{c}}}\right)$

$\psi= \begin{cases}\text { pi }-\beta-\gamma & \text { if } \quad x_{C}>x_{D} \text { and } y_{D}>y_{C} \\ \beta-\gamma & \text { if } \quad x_{D} \geq x_{C} \text { and } y_{D}>y_{C}\end{cases}$

where

$x_{C}=1_{0} \cos \left(\vartheta_{1}\right)+1_{2} \cos \left(\vartheta_{2}\right)$

$\mathrm{y}_{\mathrm{C}}=1_{0} \sin \left(\vartheta_{1}\right)+1_{2} \sin \left(\vartheta_{2}\right)$

$\mathrm{x}_{\mathrm{D}}=1_{01} \cos \left(\vartheta_{11}\right)+1_{22} \cos \left(\vartheta_{22}\right)+\mathrm{g}$

$\mathrm{y}_{\mathrm{D}}=\mathrm{l}_{01} \sin \left(\vartheta_{11}\right)+\mathrm{l}_{22} \sin \left(\vartheta_{22}\right)+\mathrm{h}_{1}$

The velocity of point $S$ can be computed through the derivative expressions of Eq. (9) by using Eqs. (10)-(12) in the form,

$\dot{\mathrm{x}}_{\mathrm{S}}=\dot{\mathrm{x}}_{\mathrm{C}}-1_{4} \dot{\psi} \sin (\psi)-1_{5} \dot{\beta} \cos (\beta)$

$\dot{\mathrm{y}}_{\mathrm{S}}=\dot{\mathrm{y}}_{\mathrm{C}}+1_{4} \dot{\psi} \cos (\psi)+1_{5} \dot{\beta} \cos (\beta)$

whose terms are computed by expressions that are given in Appendix 1.

The acceleration of point $\mathrm{S}$ can be computed through the derivative expressions of Eq. (13) to obtain,

$\ddot{\mathrm{x}}_{\mathrm{S}}=\ddot{\mathrm{x}}_{\mathrm{C}}-1_{4} \ddot{\psi} \sin \psi-1_{4} \dot{\psi}^{2} \cos \psi-1_{5} \ddot{\beta} \sin \beta-1_{5} \dot{\beta}^{2} \cos \beta$

$\ddot{y}_{S}=\ddot{y}_{C}+1_{4} \ddot{\psi} \cos \psi-1_{4} \dot{\psi}^{2} \sin \psi+1_{5} \ddot{\beta} \cos \beta-1_{5} \dot{\beta}^{2} \sin \beta$,

in which the involved derivatives can be expressed as the derivatives of the expressions in Appendix 1.

The above-mentioned analysis is also useful to compute the values of the objective functions $f_{i}$ during the optimization process.
By applying the principle of virtual power one can obtain, (Fig. 10),

$\tau \dot{\vartheta}_{1}=\mathrm{F}_{\mathrm{GA}} \mathrm{v}_{\mathrm{S}}$

in which $\tau$ indicates the actuation torque applied on the input link $1_{0}$, and $\mathrm{v}_{\mathrm{s}}$ is the velocity of contact point $\mathrm{S}$.

Finally, the Eq. (4) can be written as,

$\mathrm{IP}=\frac{1_{\text {gear }} \cos (\psi) \dot{\vartheta}_{1}}{\dot{\mathrm{y}}_{\mathrm{s}} \cos (\psi)+\dot{\mathrm{x}}_{\mathrm{s}} \sin (\psi)}$

where $\dot{\mathrm{x}}_{\mathrm{S}}$ and $\dot{\mathrm{y}}_{\mathrm{S}}$ are the components of the velocity $\mathrm{v}_{\mathrm{s}}$ in Eqs. (13). Thus Eq. (16) has been deduced from Eq. (4) by considering that $\tau=\mathrm{Ql}_{\text {gear }}$ as a torque on an input link that is given by an actuating force $\mathrm{Q}$ acting on it at a distance $1_{\text {gear, }}$ (Fig. 10).

The size L of a gripper mechanism can be given as fuction of the design parameters in the form,

$$
\mathrm{L}=\sqrt{\mathrm{1}_{0}^{2}+\mathrm{l}_{1}^{2}+\mathrm{l}_{2}^{2}+\mathrm{l}_{3}^{2}+\mathrm{l}_{4}^{2}}
$$

Results of these computations are illustrated in Figs. (11) and (12). In particular, in Fig. (11) the coupler path of point $\mathrm{S}$ has been reported together with the gripper mechanism for two-finger gripper.

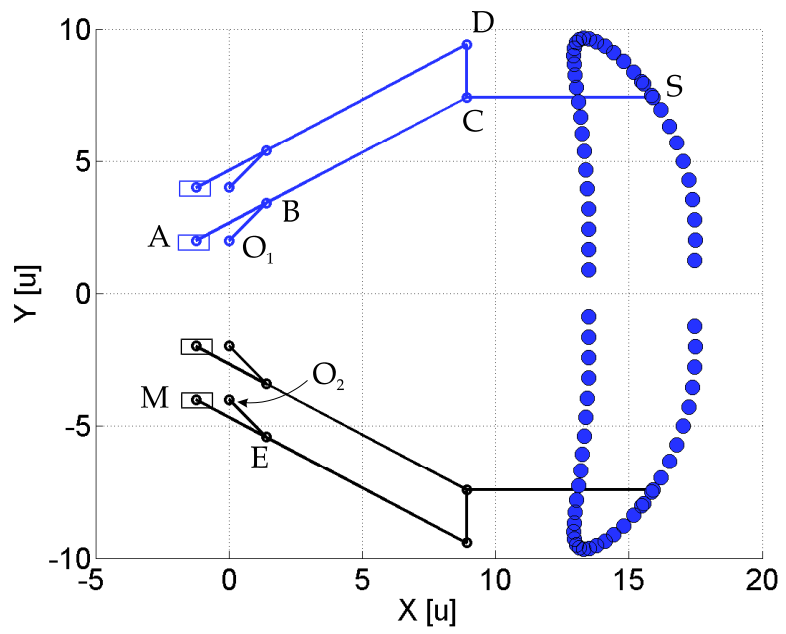

Fig. (11). A kinematic scheme of $8 P 2 R$ mechanism with path, as described by point $S$.

Fig. (12) shows kinematic characteristics of 8R2P mechanism. In particular, Fig. (12a) show the trajectory described by contact point $\mathrm{S}$ during the grasping action; Fig. (12b) shows the trajectory described by contact point $S$ with respect the input angle $\vartheta_{1}$; Fig. (12c) shows the velocity of point $\mathrm{S}$ during the grasping action; Fig. (12d) shows the acceleration of point S; Fig. (12e) shows the variation of IP with respect the input angle $\vartheta_{1},[23]$.

The displacement curves in Figs. (12b) and (12e) have been plotted with the aim to show the continuous character of the motion that starts from $\vartheta_{1}=-10 \mathrm{deg}$. up to $\vartheta_{1}=$ 190deg., according to the scheme in Fig. (10).

The above-mentioned considerations can be used in a design formulation in the form of an optimization problem. 
a)

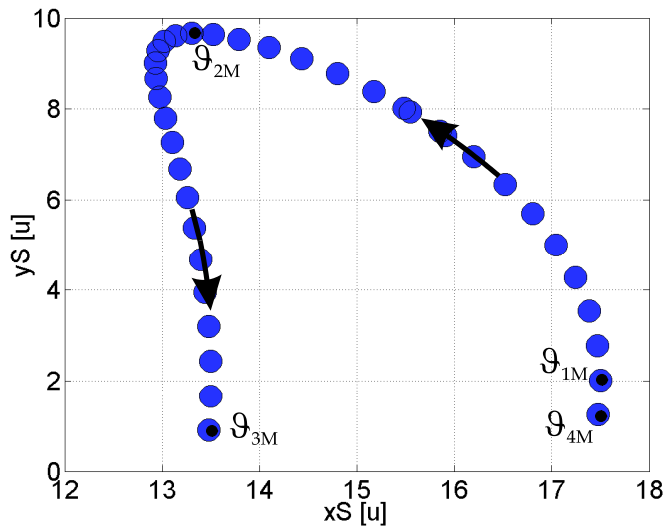

c)

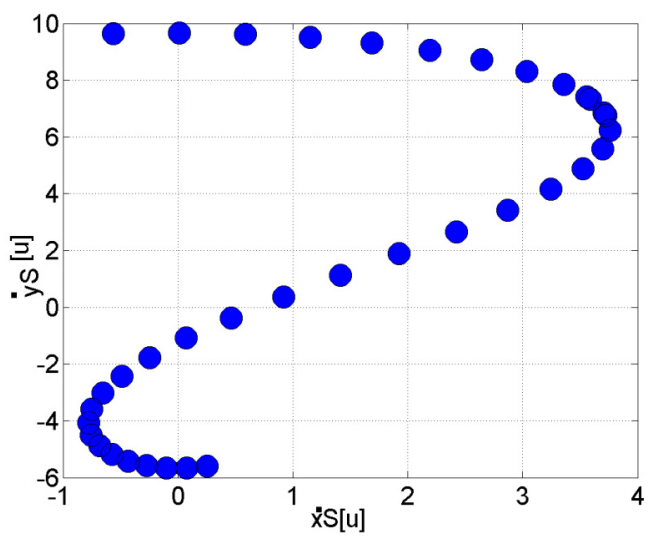

b)
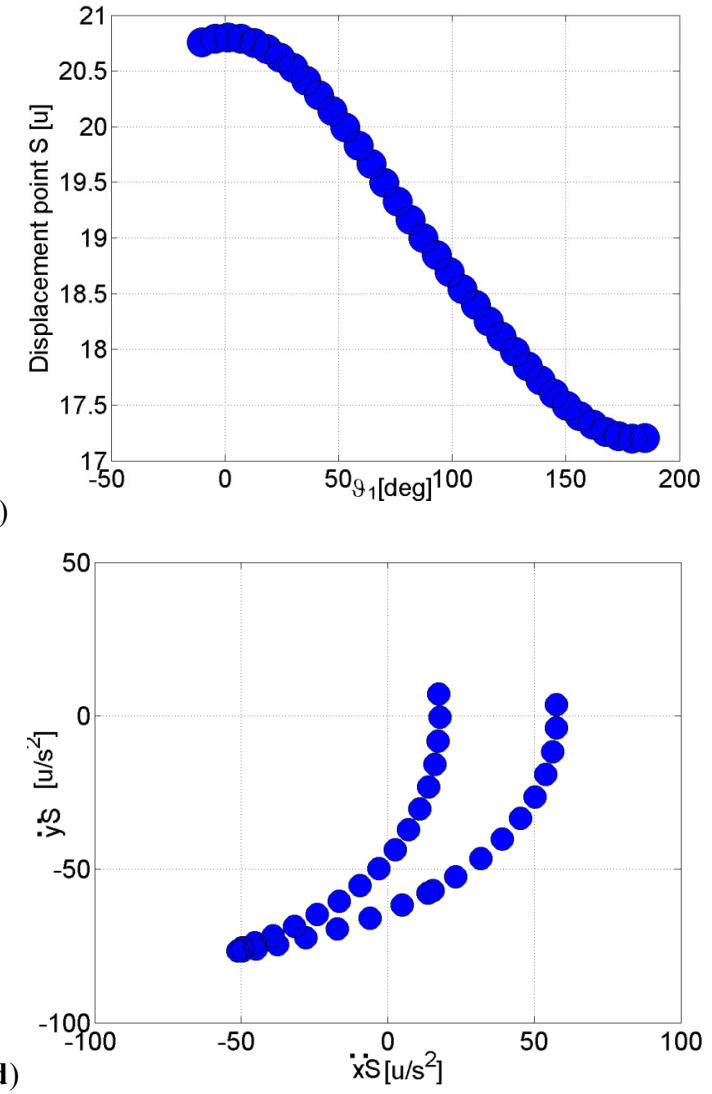

d)

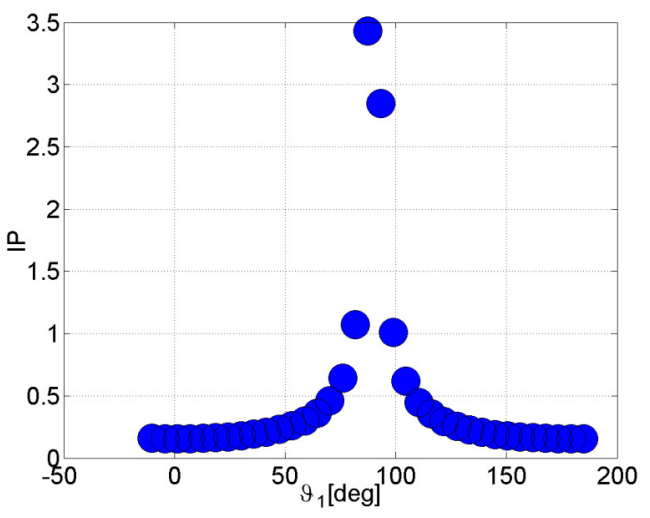

Fig. (12). Kinematic characteristics of $8 R 2 P$ mechanism in Fig. (10): a) path described by point $S$; b) displacement point $S$; c) velocity of point $S$; d) acceleration of point $S$; e) IP versus $\vartheta_{1}$.

Referring to the scheme in Fig. (10), the design parameters can be chosen as,

$\mathrm{x}_{\mathrm{k}}=\left[\mathrm{1}_{0}=\mathrm{l}_{01}, \mathrm{l}_{1}=\mathrm{l}_{11}, \mathrm{l}_{2}=\mathrm{l}_{22}, \mathrm{l}_{3}, \mathrm{l}_{4}, \mathrm{~h}_{1}, \mathrm{~h}_{2}=\mathrm{h}_{3}=\mathrm{g}=0\right.$,

$\left.\gamma=\pi / 2,1_{5}=0.1,1_{\text {gear }}=1.4\right]$

In order to consider the above-mentioned requirements and constrains and find an optimum solution in the whole range of objects that can be grasped, the optimisation design problem can be defined as,

$\min \mathbf{F}(\mathbf{x})$

subject to,

$\mathrm{g}_{1}=\operatorname{Min} X_{\mathrm{S}}-\min X_{\mathrm{S} 0} \geq 0$

$\mathrm{g}_{2}=\operatorname{Min} \mathrm{Y}_{\mathrm{S}}-\min \mathrm{Y}_{\mathrm{S} 0} \geq 0$
$\mathrm{g}_{3}=\operatorname{Max} \mathrm{X}_{\mathrm{S}}-\max \mathrm{X}_{\mathrm{S} 0} \leq 0$

$\mathrm{g}_{4}=\operatorname{Max} \mathrm{Y}_{\mathrm{S}}-\max \mathrm{Y}_{\mathrm{S} 0} \leq 0$

where $F_{i}=f_{i}$ is the $i$-th component of the vector $\mathbf{F}$ as a function of the design variables in vector $\mathbf{x}$.

The objective functions $f_{i}(i=1,2,3,4)$ have been expressed as a synthetic criterion of the grasping requirements for a grasping mechanism. IP can be computed for each possible configuration of grasping mechanism. The design constraints defined in Eqs. (20)-(23) are expressed in term of the area that can be reached by the contact point S. If the mechanism is symmetric only one finger mechanism can be analysed, and this area can be defined by the given coordinates $\min X_{\mathrm{S} 0}, \min _{\mathrm{S} 0}, \max _{\mathrm{S} 0}$ and $\max _{\mathrm{S} 0}$ of the 
generic contact point $\mathrm{S}$ in a fixed frame as shown in Fig. (13).

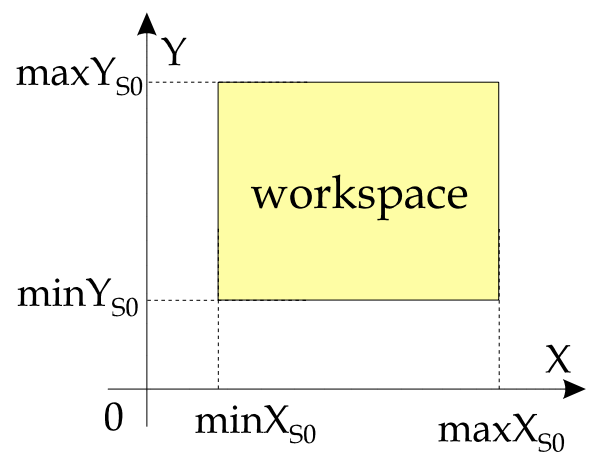

Fig. (13). Workspace area for a contact point $S$.

The numerical procedure, which has been adopted in this work, is summarized in the flowchart in Fig. (14) where the utilization of the proposed formulation has been emphasized as well as a Sequential Quadratic Programming procedure for the non linear design problem. Indeed, the Optimization Toolbox of Matlab [32] has been used to perform the numerical solution with a Sequential Quadratic Programming procedure. This numerical procedure works in such a way that at each step $\mathrm{k}$ a solution is found along a search direction $\delta_{\mathrm{k}}$ with a variable update $\psi_{\mathrm{k}}$. The iteration continues until the objective vectors converge.

The procedure has been developed so that the formulation has been easily included within the solving procedure for the optimization problem by using the facilities of the Optimization Toolbox of Matlab, [32], which permits an easy arrangement for an optimum design with analytical expressions.

Once the numerical computations are convergent to a feasible solution, the velocity and the accuracy of the solution can be enhanced by the designer by updating the convergence parameters $\varepsilon_{\mathrm{f}}$ and $\varepsilon_{\mathrm{g}}$, which refer to the multiobjective functions $f_{i}(i=1,2,3,4)$ in Eq. (19) and the constraints functions $\mathrm{g}_{\mathrm{i}}(\mathrm{i}=1,2,3,4)$ in Eqs. (20)-(23), respectively.

Eqs. (9) to (17) have been used in order to optimize the design procedure developed by using the routine "minimax" of Matlab Optimization Toolbox, [32], by considering the objective functions expressed by Eq. (19) and constraints expressed by Eqs. (20) to (23), [23].

The design parameters have chosen as $1_{0}=1_{01}=1.8 \mathrm{u}, 1_{1}=$ $1_{11}=3.0 \mathrm{u}, 1_{2}=1_{22}=12.0 \mathrm{u}, 1_{3}=1.4 \mathrm{u}, 1_{4}=7.0 \mathrm{u}, 1_{5}=0.1 \mathrm{u}$,

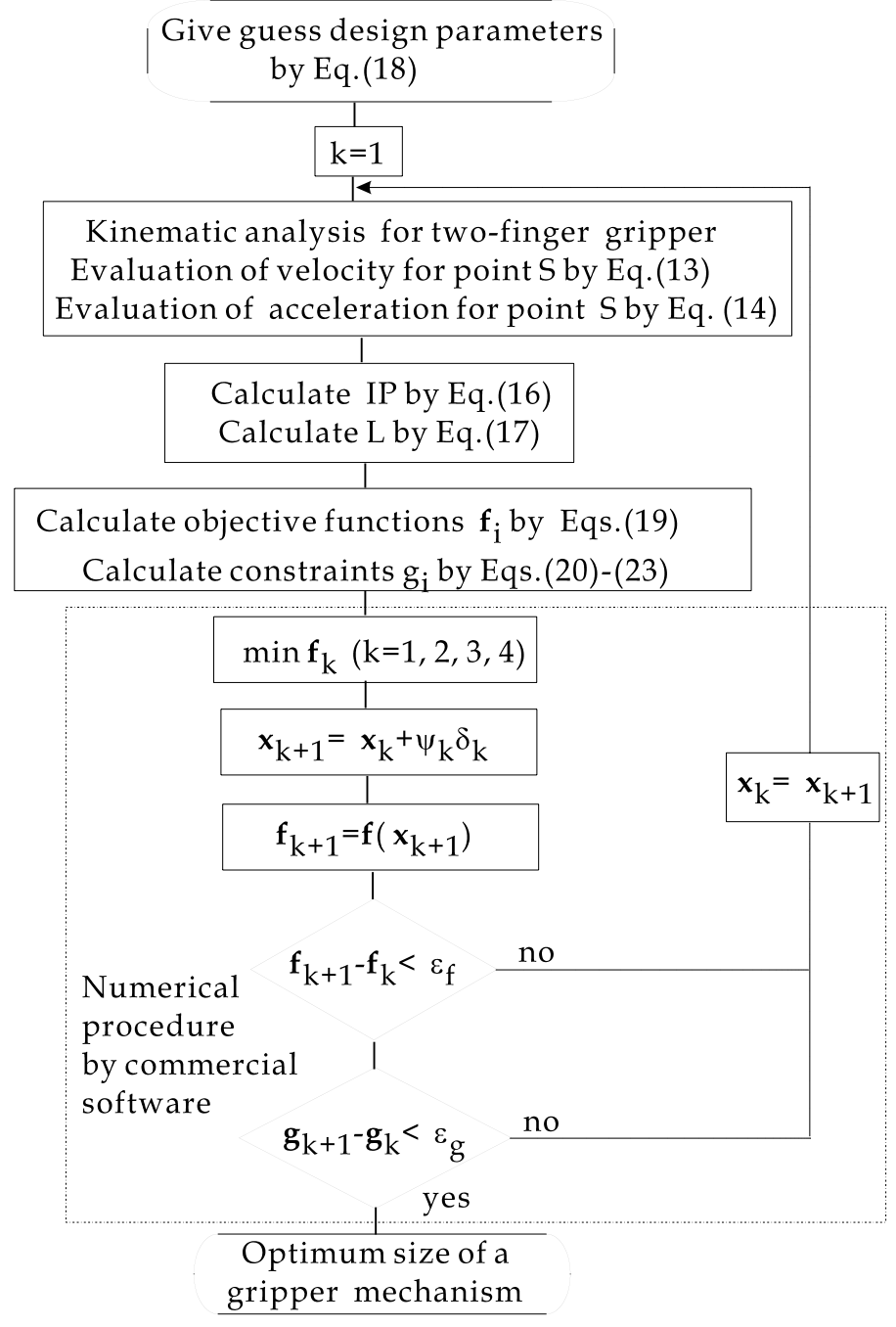

Fig. (14). Flowchart of a numerical procedure for optimum design of two-finger gripper mechanism. 
$\mathrm{h}_{1}=1.4 \mathrm{u}, \mathrm{h}_{2}=\mathrm{h}_{3}=\mathrm{g}=0 \mathrm{u}, \gamma=\pi / 2$ deg., $1_{\text {gear }}=1.4 \mathrm{u}$ (Lengths are expressed in $u$ unit and angles in degrees).

Referring to Fig. (15), the inequality constraints have been chosen, referring to Eqs. (20)-(23) as $\min X_{\mathrm{S} 0}=18 \mathrm{u}$, $\min _{\mathrm{S} 0}=-4.5 \mathrm{u}, \operatorname{maxX}_{\mathrm{S} 0}=20.5 \mathrm{u}$ e $\max _{\mathrm{S} 0}=8 \mathrm{u}$, respectively. The prescribed workspace has been chosen with an asymmetric area with the aim to stress how the algorithm can give optimal solution with suitable symmetrical workspace even when the starting guess could be not suitably assigned.

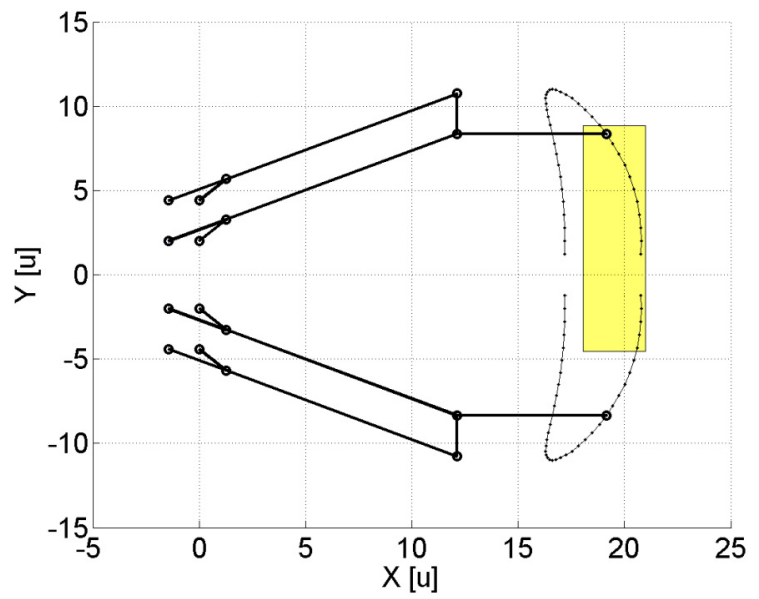

Fig. (15). A prescribed working area.

The results of the proposed case of study are shown in the plots of Figs. (16) to (19).

a)
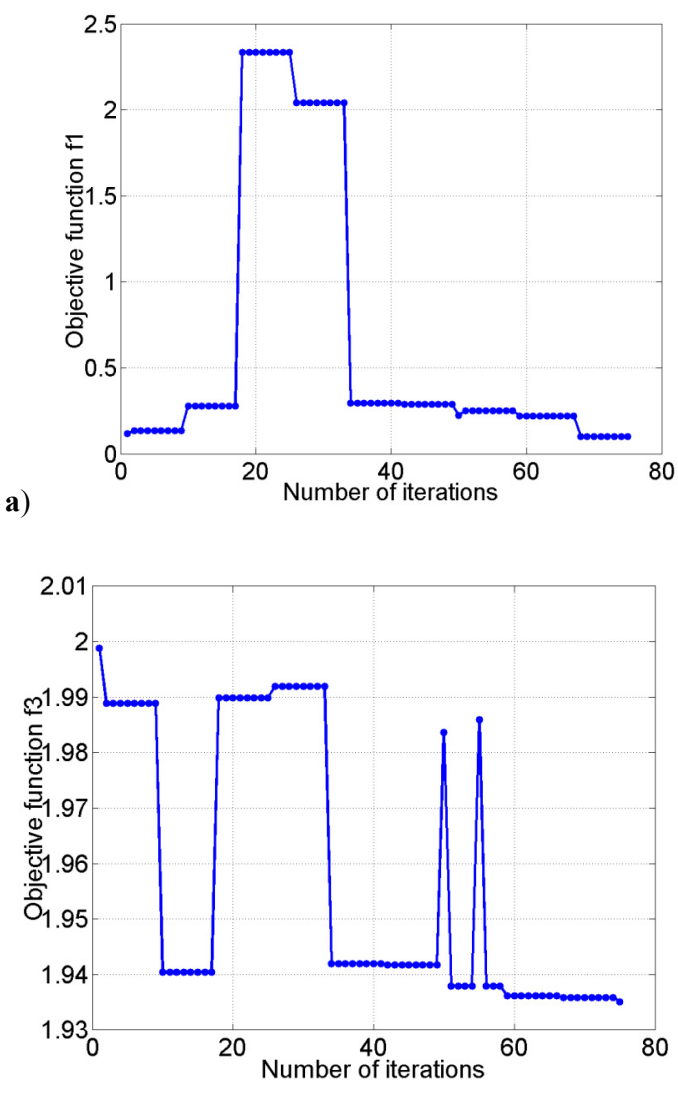

In particular, Fig. (16) show the evolution of the multiobjective functions versus the number of iteration. It is worth noting that the optimization algorithm has converged very rapidly to an optimum solution in 76 iterations as reported in Table 1.

Table 1. Optimal Values of Objective Functions

\begin{tabular}{|c|c|c|c|c|c|}
\hline Solution & $\mathbf{f}_{\mathbf{1}}$ & $\mathbf{f}_{\mathbf{2}}$ & $\mathbf{f}_{\mathbf{3}}$ & $\mathbf{f}_{\mathbf{4}}$ & $\begin{array}{c}\mathbf{n r .} \\
\text { iteration }\end{array}$ \\
\hline \hline Guess & 0.117 & 14.40 & 2.00 & 0.99 & - \\
\hline Optimal & 0.101 & 14.13 & 1.93 & 0.86 & 76 \\
\hline
\end{tabular}

Fig. (17) shows the evolution of the design parameters versus the number of iteration, whereas Table 2 shows the numerical results.

Table 2. Optimal Values of Design Parameters

\begin{tabular}{|c|c|c|c|c|c|c|}
\hline Solution & $\begin{array}{c}\mathbf{l}_{\mathbf{0}}=\mathbf{l}_{\mathbf{0 1}} \\
{[\mathbf{u}]}\end{array}$ & $\begin{array}{c}\mathbf{l}_{\mathbf{1}}=\mathbf{l}_{\mathbf{1 1}} \\
{[\mathbf{u}]}\end{array}$ & $\begin{array}{c}\mathbf{l}_{\mathbf{2}}=\mathbf{I}_{\mathbf{2 2}} \\
{[\mathbf{u}]}\end{array}$ & $\begin{array}{c}\mathbf{l}_{\mathbf{3}} \\
{[\mathbf{u}]}\end{array}$ & $\begin{array}{c}\mathbf{l}_{\mathbf{4}} \\
{[\mathbf{u}]}\end{array}$ & $\begin{array}{c}\mathbf{h}_{\mathbf{1}} \\
{[\mathbf{u}]}\end{array}$ \\
\hline \hline Guess & 1.80 & 3.00 & 12.00 & 1.40 & 7.00 & 1.40 \\
\hline Optimal & 1.23 & 3.23 & 9.71 & 1.59 & 9.52 & 1.28 \\
\hline
\end{tabular}

In particular, Fig. (17a) shows the evolution of the design parameters $l_{0}, l_{1}$ and $l_{2}$, whereas Fig. (17b) shows the evolution of the design parameters of $l_{3}, l_{4}$ and $h_{1}$.

b)
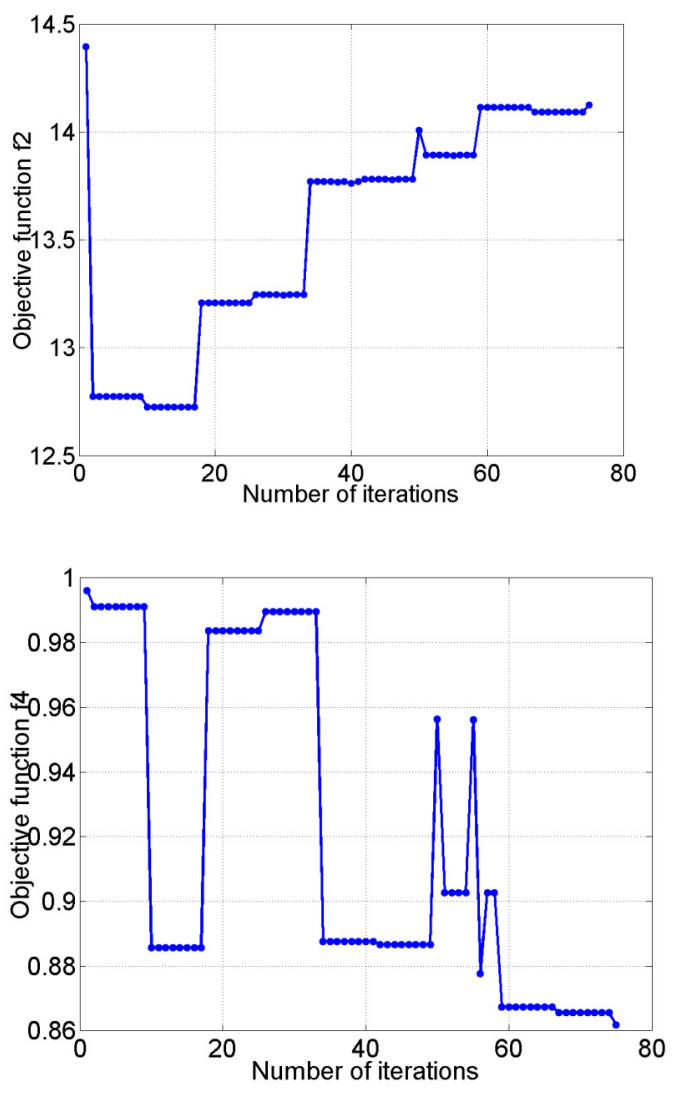

Fig. (16). Evolution of the objective functions versus number of iterations for the case of study in Table $1:$ a) $f_{1} ;$ b) $f_{2} ;$ c) $f_{3} ;$ d) $f_{4}$. 
a)

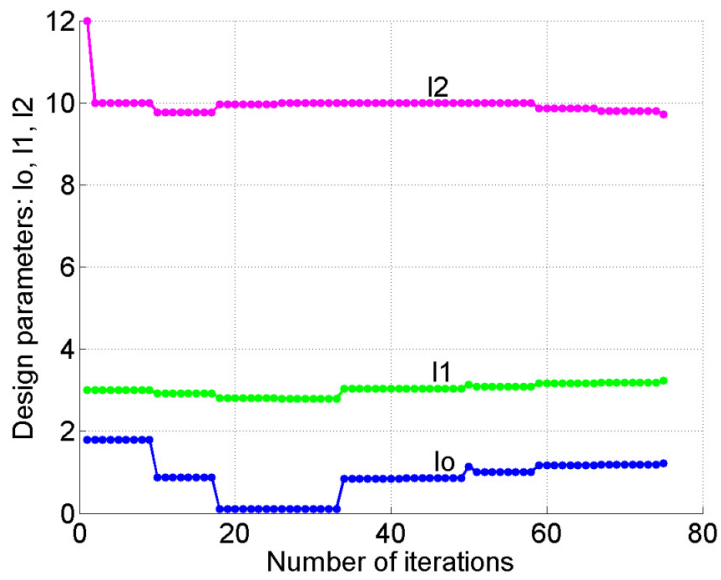

b)

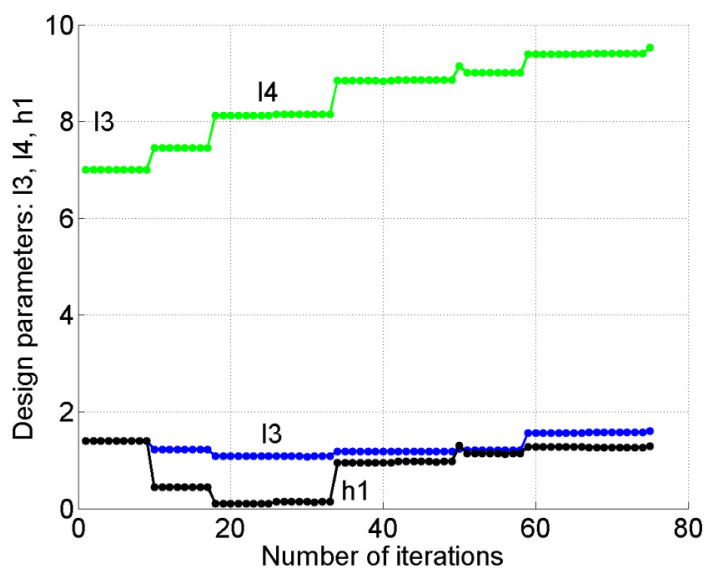

Fig. (17). Evolution of design parameters versus number of iterations: a) $1_{0}, 1_{1}$ and $1_{2}$; b) $1_{3}, 1_{4}$ and $h_{1}$.

Fig. (18) shows the evolution of the constraints versus the number of iteration, whereas Table $\mathbf{3}$ shows the numerical results. In particular, Fig. (18a) shows the evolution of significant constraints $g_{1}, g_{2}$, whereas Fig. (18b) shows the evolution of significant constraints $g_{3}$ and $g_{4}$.

Table 3. Optimal Values Constraints

\begin{tabular}{|c|c|c|c|c|}
\hline Constraints & $\begin{array}{c}\mathbf{g}_{1} \\
{[\mathbf{u}]}\end{array}$ & $\begin{array}{c}\mathbf{g}_{2} \\
{[\mathbf{u}]}\end{array}$ & $\begin{array}{c}\mathbf{g}_{3} \\
{[\mathbf{u}]}\end{array}$ & $\begin{array}{c}\mathbf{g}_{4} \\
{[\mathbf{u}]}\end{array}$ \\
\hline \hline Initial & 2.15 & 1.90 & 0.30 & 2.57 \\
\hline Optimal & 0 & 0 & -0.04 & -2.71 \\
\hline
\end{tabular}

In particular, Fig. (18a) shows the evolution of two significant constraints $\mathrm{g}_{1}$ and $\mathrm{g}_{2}$ during the optimization process to illustrate how fast and accurately the constraints have been satisfied. Similarly, Fig. (18b) shows the evolution of two significant constraints $\mathrm{g}_{3}$ and $\mathrm{g}_{4}$ during the optimization process. In particular, it has been shown how fast and accurately constraint $\mathrm{g}_{3}$ has been satisfied.

Fig. (19) shows the optimum kinematic chain obtained by considering the optimum design parameters listed in Table $\mathbf{1}$.

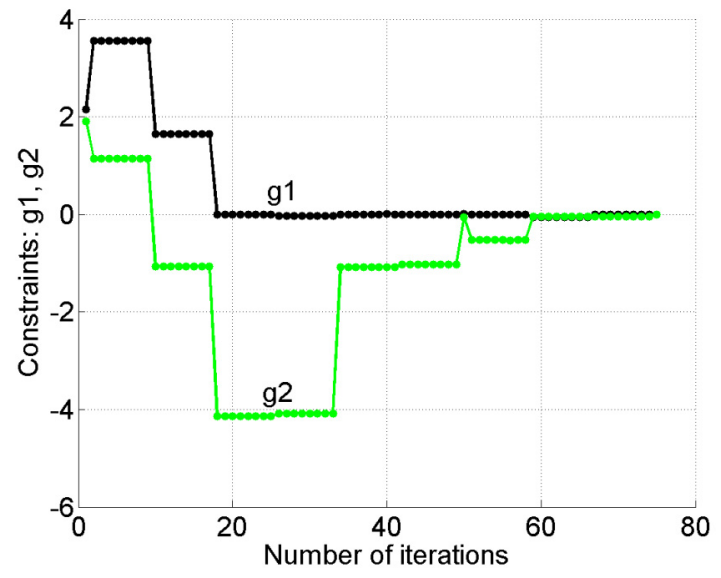

In particular, Fig. (19a) shows the path described by contact point S. Fig. (19b) shows the related obtained working area with respect the imposed constraints sketched by a rectangle, which shows how accurately constraints have been satisfied.

The optimal grasping mechanism satisfies requirements and constraints and the given working area.

\section{CONCLUSIONS}

In this paper, a review of existing industrial two-finger grippers has been used to formulate an optimum design procedure for two-finger gripper mechanisms as based on main characteristics of gripper operation.

An original multi-objective optimum algorithm has been used by considering four different objective functions, namely grasping index, encumbrance of mechanism, acceleration and velocity for fingers with respect to a prescribed working area.

This new formulation will achieve a kinematic design of gripper mechanism with optimal characteristics even as improvement of existing solutions.

A case of study has been presented for designing a gripper mechanism with an 8R2P linkage. The newly design

b)

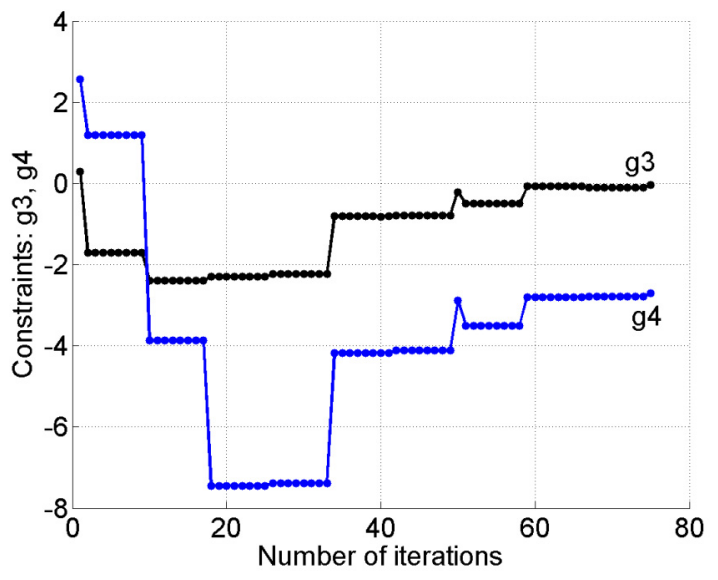

Fig. (18). Evolution of constraints versus number of iterations: a) $g_{1}$ and $g_{2} ;$ b) $g_{3}$ and $g_{4}$. 
a)

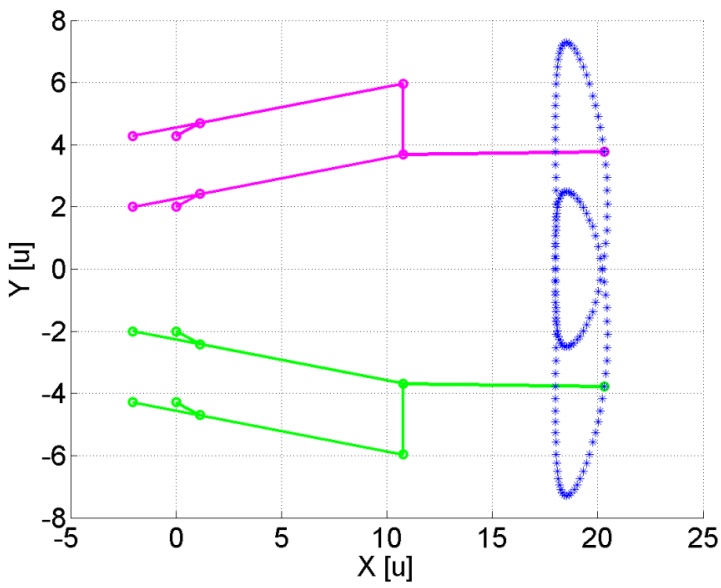

b)

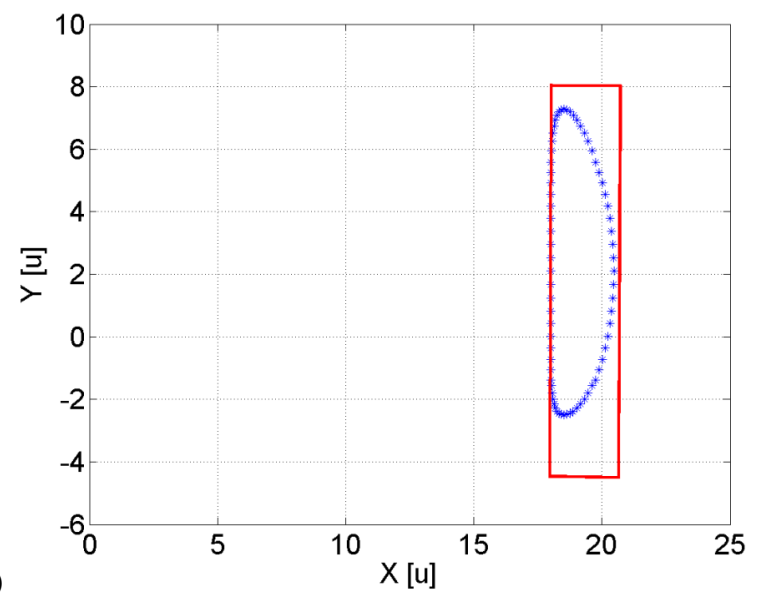

Fig. (19). Optimum results for the 8R2P mechanism: a) chain; b) working area with respect the prescribed one.

mechanism show more compact structure and optimal operation features as compared with the guess industrial example.

Numerical results have been reported to show the soundness of the proposed new optimum design procedure by referring to computational and practical results.

\section{APPENDIX 1}

$\dot{\mathrm{x}}_{\mathrm{C}}=-\mathrm{1}_{0} \dot{\vartheta}_{1} \sin \left(\vartheta_{1}\right)-1_{2} \dot{\vartheta}_{2} \sin \left(\vartheta_{2}\right)$

$\dot{\mathrm{y}}_{\mathrm{C}}=1_{0} \dot{\vartheta}_{1} \cos \left(\vartheta_{1}\right)+1_{2} \dot{\vartheta}_{2} \cos \left(\vartheta_{2}\right)$

$\dot{\vartheta}_{2}=\frac{1_{0} \dot{\vartheta}_{1} \cos \left(\vartheta_{1}\right)}{1_{1} \cos \left(\vartheta_{2}\right)}, \quad \dot{\beta}=C_{1} \dot{\vartheta}_{1}+C_{2} \dot{\vartheta}_{11}$

$\dot{\vartheta}_{11}=\left(\frac{A-C_{1} 1_{3} \sin (\beta)}{A_{1}+C_{2} 1_{3} \sin (\beta)}\right) \dot{\vartheta}_{1}$

$\dot{\psi}=\left\{\begin{array}{llll}-\dot{\beta} & \text { if } \quad x_{C}>x_{D} \quad \text { and } & y_{D}>y_{C} \\ \dot{\beta} & \text { if } \quad x_{D} \geq x_{C} \quad \text { and } & y_{D}>y_{C}\end{array}\right.$

$C_{1}=\frac{-B\left(x_{D}-x_{C}\right)+\left(y_{D}-y_{C}\right) A}{\left(x_{D}-x_{C}\right)^{2}+\left(y_{D}-y_{C}\right)^{2}}$

$C_{2}=\frac{B_{1}\left(x_{D}-x_{C}\right)-\left(y_{D}-y_{C}\right) A_{1}}{\left(x_{D}-x_{C}\right)^{2}+\left(y_{D}-y_{C}\right)^{2}}$

$A=\left\{-1_{0} \sin \left(\vartheta_{1}\right)-\frac{1_{0} 1_{2} \cos \left(\vartheta_{1}\right) \sin \left(\vartheta_{2}\right)}{1_{1} \cos \left(\vartheta_{2}\right)}\right\}$

$\mathrm{B}=\left\{\frac{\mathrm{l}_{0} \mathrm{l}_{2} \cos \left(\vartheta_{1}\right)}{\mathrm{l}_{1}}+\mathrm{l}_{0} \cos \left(\vartheta_{1}\right)\right\}$

$A_{1}=\left\{-1_{01} \sin \left(\vartheta_{11}\right)-\frac{1_{01} 1_{22} \cos \left(\vartheta_{11}\right) \sin \left(\vartheta_{22}\right)}{1_{11} \cos \left(\vartheta_{22}\right)}\right\}$

$$
\begin{aligned}
& \mathrm{B}_{1}=\left\{\frac{\mathrm{l}_{01} \mathrm{l}_{22} \cos \left(\vartheta_{11}\right)}{\mathrm{l}_{11}}+\mathrm{l}_{01} \cos \left(\vartheta_{11}\right)\right\} \\
& \ddot{x}_{C}=-1_{0} \ddot{\vartheta}_{1} \sin \vartheta_{1}-1_{0} \dot{\vartheta}_{1}^{2} \cos \vartheta_{1}-1_{2} \ddot{\vartheta}_{2} \sin \vartheta_{2}-1_{2} \dot{\vartheta}_{2}^{2} \cos \vartheta_{2} \\
& \ddot{y}_{C}=1_{0} \ddot{\vartheta}_{1} \cos \vartheta_{1}-1_{0} \dot{\vartheta}_{1}^{2} \sin \vartheta_{1}+1_{2} \ddot{\vartheta}_{2} \cos \vartheta_{2}-1_{2} \dot{\vartheta}_{2}^{2} \sin \vartheta_{2} \\
& \ddot{x}_{D}=-1_{01} \ddot{\vartheta}_{11} \sin \vartheta_{11}-1_{01} \dot{\vartheta}_{11}^{2} \cos \vartheta_{11}-1_{22} \ddot{\vartheta}_{22} \sin \vartheta_{22}+ \\
& -1_{22} \dot{\vartheta}_{22}^{2} \cos \vartheta_{22} \\
& \ddot{y}_{\mathrm{D}}=1_{01} \ddot{\vartheta}_{11} \cos \vartheta_{11}-1_{01} \dot{\vartheta}_{11}^{2} \sin \vartheta_{11}+1_{22} \ddot{\vartheta}_{22} \cos \vartheta_{22}+ \\
& -1_{22} \dot{\vartheta}_{22}^{2} \sin \vartheta_{22} \\
& \ddot{\vartheta}_{2}=\frac{1_{0} \ddot{\vartheta}_{1} \cos \vartheta_{1}-1_{0} \dot{\vartheta}_{1}^{2} \sin \vartheta_{1}+1_{1} \dot{\vartheta}_{2}^{2} \sin \vartheta_{2}}{1_{1} \cos \vartheta_{2}} \\
& \dot{\vartheta}_{22}=\frac{1_{01} \ddot{\vartheta}_{11} \cos \vartheta_{11}-1_{01} \dot{\vartheta}_{11}^{2} \sin \vartheta_{11}+1_{11} \dot{\vartheta}_{22}^{2} \sin \vartheta_{22}}{1_{11} \cos \vartheta_{22}} \\
& \ddot{\beta}=\frac{-1_{3} \dot{\beta}^{2} \cos \beta-\ddot{x}_{D}+\ddot{x}_{C}}{1_{3} \cos \beta} \\
& \ddot{\vartheta}_{11}=\frac{1_{22}}{1_{0}} \ddot{\vartheta}_{22} \cos \left(\vartheta_{11}+\vartheta_{22}\right)-\frac{1_{22}}{1_{0}} \ddot{\vartheta}_{11} \sin \left(\vartheta_{22}-\vartheta_{11}\right)
\end{aligned}
$$

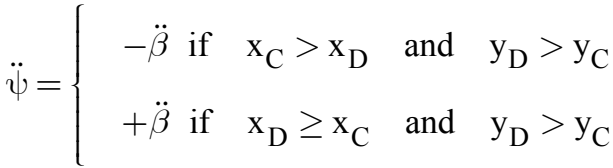

\section{REFERENCES}

[1] G. Lundstrom, "Industrial robot grippers", The Industrial Robot, Vol. 1, pp. 72-82, 1973.

[2] F. Y. Chen, "Gripping mechanisms for industrial robots", Mechanism Machine Theory, Vol. 17, pp. 299-311, 1982.

[3] S. Y. Nof, Handbook of industrial robotics: New York: Editorial Wiley, 1985. 
[4] G. Belforte, "Industrial robot gripper", Notiziario Tecnico AMMA, Torino, 128-136, 1985.

[5] D. T. Pham, and W. B. Heginbotham, Robot grippers, Bedford: IFS Publications Limited, 1986.

[6] R. D. Potter, "End-of-arm tooling", in Handbook of Industrial Robotics, S. Y. Nof, Ed, New York: Wiley, 1985, pp. 775-787.

[7] M. Ceccarelli, "Design problems for industrial robot two-finger grippers", in Proceedings of the $4^{\text {th }}$ International Workshop on Robotics in Alpe-Adria Region, Bled, 1994, pp. 568-571.

[8] I. B. Chelpanov, and S. N. Kolpashnikov, "Problems with the mechanics of industrial robot grippers", Mechanism and Machine Theory, pp. 295-299, 1983.

[9] N. P. Belfiore, and E. Pennestrì, "An atlas of linkage-type robotic grippers", Mechanism and Machine Theory, Vol. 32, (n. 7), pp. 811-833, 1997.

[10] F. Lhote, J. M. Kauffmann, P. Andrè, and J. P. Taillard, in Robot Technology: Robot Components and Systems, Vol. 4, Chapter 8: End Effectors, Kogan Page, London, 1987, pp. 297-312.

[11] S. N. Dwivedi, S. C. Sharma, and M. Sharifi, "Design of an intelligent gripper for space application" in The $8^{\text {th }}$ World Congress on the Theory of Machines and Mechanisms, Prague, 1991, pp. 453-456.

[12] K. B. Shimoga, "Robot grasp synthesis algorithms: a survey", International Journal of Robotics Research, Vol. 4, (n. 4), pp. 3-17, 1986.

[13] B. Salunkhe, W. X. Mao, and U. Tasch, "Optimal grasping formulations that result in high quality and robust configurations", Journal of Robotic Systems, Vol. 15, (n. 12), pp. 713-729, 1998.

[14] V. N. Dubey, R. M. Crowder, and P. H. Chappell, "Optimal object grasp using tactile sensors and fuzzy logic", Robotica, Vol. 17, (n. 6), pp. 685-693, 1999.

[15] R. D. Hester, M. Cetin, C. Kapoor, and D. Tesar, "A criteria-based approach to grasp synthesis", in Proceedings of the 1999 IEEE International Conference on Robotics \& Automation, Detroit, 1999, pp. 1255-1260.

[16] A. Osyczka and S. Krenich, "A new constraint tournament selection method for multicriteria optimization using genetic algorithm", in Proceedings of the Congress of Evolutionary Computing, San Diego, 2000, pp. 501-509.

[17] O. H. Penisi, G. Carbone, and M. Ceccarelli, "Optimum design and testing of mechanisms for two-finger gripper", in Proceedings of
MUSME2002, The International Symposium on Multibody Systems and Mechatronics, Mexico City, 2002, Paper n. M03.

[18] M. Ceccarelli, J. Cuadrado, and D. Dopico, "An optimum synthesis for gripping mechanisms by using natural coordinates", Journal of mechanical Engineering Science, IMechE 2002, Vol. 216, Part C, pp. 643-653, 2002.

[19] S. Krenich, "Multicriteria design optimization of robot gripper mechanisms", in IUTAM Symposium on Evolutionary Methods in Mechanics, Proceedings of the IUTAM Symposium, 2002, Vol. 117, pp. 207-218.

[20] M. Ceccarelli, "An optimum design for a gripping mechanism", in Proceedings of the International Symposium Machines and Mechanisms ISMM'97, Beograd, 1997, pp. 23-26.

[21] M. Ceccarelli, Ed., in Fundamentals of mechanics of robotic manipulation, Dordrecht: Kluwer Academic Publisher, 2004.

[22] Y. Zheng, and W. H. Qian, "Limiting and minimizing the contact forces in multifingered grasping", Mechanism and Machine Theory, Vol. 41, pp. 1247-1257, 2006.

[23] C. Lanni, "A characterization of impact grasping and optimum design of gripper mechanisms", PhD thesis, LARM, University of Cassino, Cassino, Italy, 2008.

[24] Gimatic Handling, 2008. Available from: www.gimatic.com

[25] GMG System, 2008. Available from: http://www.gmgsystem.com/en/fr_home. html

[26] AGI Components, 2008. Available from: www.agiautomation.com/index.html

[27] SMC Components, 2008. Available from: www.smcworld.com/ 2002 / e / webcatalog / docs / actuator / gripper / gripper. html

[28] S. S. Rao, "Multicriteria optimization in mechanisms", Transaction of ASME, Journal of Mechanical Design, Vol. 101, pp. 398-406, 1985.

[29] K. Tanie, "Design of robot hands" in Handbook of Industrial Robotics, S. Y. Nof, Ed, New York: Wiley, 1985, pp. 112-137.

[30] IBM 7565, IBM 7565 manufacturing system, IBM Hardware Library, 1985.

[31] M. Ceccarelli, and J. Nieto, "Kinematic evaluation of a robotic gripper", Anales de Ingenieria Mecanica, Año 6, Vol. 1, pp. 159164, 1988.

[32] A. Grace, Optimization Toolbox for use with matlab, Natick: The Math Works Inc., 1992.

(C) Lanni and Ceccarelli; Licensee Bentham Open.

This is an open access article licensed under the terms of the Creative Commons Attribution Non-Commercial License (http: //creativecommons.org/licenses/by$\mathrm{nc} / 3.0 /$ ), which permits unrestricted, non-commercial use, distribution and reproduction in any medium, provided the work is properly cited. 